\title{
Interleukin-15 as a potential regulator of the innate immune response
}

W. Carson ${ }^{1}$ and M.A. Caligiuri ${ }^{2}$

\author{
Divisions of ${ }^{1}$ Surgery and ${ }^{2}$ Medicine, Roswell Park Cancer Institute,
} Buffalo, NY, USA
Correspondence

M.A. Caligiuri

The Arthur James Comprehensive

Cancer Center

Division of Hematology-Oncology

458A Starling Loving Hall

320 W. 10th Ave.

Columbus, $\mathrm{OH} 43210$

Fax: (614) 293-7522

E-mail: caligiuri-1@medctr.osu.edu

Presented at the International Meeting on Cytokines, Angra dos Reis, RJ, Brasil, November 24-28, 1996.

Received September 4, 1997

Accepted September 24, 1997

\section{Abstract}

Interleukin-15 (IL-15) is a newly-discovered cytokine that is produced by activated monocytes early in the course of the innate immune response. IL-15 is able to bind to components of the interleukin-2 receptor (IL-2R) despite the fact that it has no sequence homology with IL-2. IL-15 stimulates human natural killer cell proliferation, cytotoxicity, and cytokine production and can substitute for IL-2 under most conditions. In vitro studies indicate that monocyte-derived IL-15 may be an important determinant of IFN-gamma production by NK cells. In addition, IL-15 is able to promote the survival of natural killer cells under serum-free conditions. The IL-15 receptor is a heterotrimeric complex which is composed of the IL-2R $\beta$ and $\gamma$ chains in combination with a unique alpha chain (IL-15 $\alpha$ ). The IL-15R $\alpha$ chain has strong sequence homology to the IL-2R $\alpha$ chain and confers high affinity binding to the IL-15R. In contrast to IL-2, transcript for IL-15 and IL-15 $\alpha$ is expressed in a number of tissues and indicates that IL-15 may be an important ligand for cells that express components of the IL-2R.

\section{Introduction}

Interleukin-15 (IL-15) is a cytokine which binds to the $\beta$ and $\gamma$ subunits of the interleukin-2 receptor (IL-2R) and can activate cells which bear functional forms of this receptor. Like IL-12, IL-15 is secreted by activated monocytes and helps regulate the production of interferon-gamma (IFN- $\gamma$ ) by human natural killer (NK) cells. IL-15 may therefore prove to be an important regulator of the innate immune response to infection. In addition, the transcript for IL-15 is expressed in a number of normal tissues, including liver, kidney, and muscle. IL-15 may therefore prove to be an important ligand for cells that express the IL-2R.
Key words

- Interleukin-15

- Interleukin-12

- Gamma interferon

- Natural killer cell

- Innate immunity

\section{Discovery}

Grabstein et al. (1) analyzed conditioned media from a panel of cell lines for the ability to stimulate the proliferation of the IL-2-dependent cell line CTLL. 2 in the presence of a neutralizing IL-2 monoclonal antibody (mAb). A 14-kDa protein was purified from the supernatants of the simian kidney epithelial cell line CV-1 EBNA which stimulated the proliferation of CTLL.2 cells. A full-length IL-15 cDNA was subsequently isolated using the PCR reaction and degenerate oligonucleotide primers. Analysis of the amino acid sequence coded by the cloned cDNA revealed a protein of 114 amino acids. IL-15 exhibits no amino acid sequence 
homology to IL-2 or any other members of the cytokine family. In fact, IL-15 is unrelated to any known protein within the GenBank database (1). However, IL-15 is able to activate cells via components of the IL-2R.

Analysis of the predicted secondary structure of this protein reveals the presence of strong helical moments at residues 1-17 and 94-112. Two disulfide bonds are also present, one of which is analogous to the single disulfide cross-link $\left(\mathrm{Cys}^{42}-\mathrm{Cys}^{88}\right)$ in IL-2. IL-2 is classified as a 4-helix bundle cytokine and computer analysis of the IL-15 amino acid sequence predicts a 4-helix structure for this cytokine as well (1). Human IL-15 has since been cloned from a bone marrow stromal cell line, and comparison of the human and simian IL-15 cDNAs reveals $97 \%$ sequence homology within their coding regions (2).

Anderson et al. (3) have analyzed the location and organization of the IL-15 gene in the mouse. The murine IL-15 gene locus is approximately 34 kilobases in length and contains 7 introns and 8 exons. Exons 2, 4, 5 , 6,7 , and 8 code for the mature protein. In humans, IL-15 is present as a single copy gene on chromosome 4 at band $\mathrm{q} 31$ and appears to possess the same structure and organization as its murine counterpart (Mendrola $\mathrm{J}$ and Huebner K, personal communication). This region of chromosome 4 also encodes the genes for IL-2, IL-8, epidermal growth factor, and fibroblast growth factor. Despite their relative proximity, there is no evolutionary evidence to suggest that the gene for IL-15 is derived from the IL-2 locus (3).

\section{Expression}

Analysis of human tissues reveals that IL-15 mRNA is abundantly expressed in skeletal muscle as well as in the placenta. It is also expressed to a lesser extent in heart, lung, liver, kidney, and pancreas (1). Transcript for IL-15 is also highly expressed by adherent peripheral blood mononuclear cells (PBMC; i.e., CD14 ${ }^{+}$monocytes) which have been stimulated with lipopolysaccharide (LPS), the carbohydrate portion of the cell wall of Gram-negative bacteria (1). This finding has been confirmed at the protein level in purified monocytes following $5 \mathrm{~h}$ of stimulation with LPS (4), and in cultured bone marrow stromal cells (5). In contrast, fresh peripheral blood mononuclear cells express very little if any IL-15 protein (1). T cells do not express any detectable IL-15 transcript in either the resting or activated state, but IL-15 transcript is expressed by several transformed $\mathrm{T}$ cell lines $(1,6)$.

IL-15 stimulates cells via components of the IL-2 receptor

IL-2 has been used to generate lymphokine-activated killer cells (LAK) from PBMC and IL-2 is also critical to the induction of alloantigen-specific cytotoxic $\mathrm{T}$ lymphocytes (CTL) in mixed lymphocyte cultures (1). Interestingly, culture of PBMC in IL-15 gave rise to LAK cells which were slightly superior to those obtained in the presence of IL-2, especially when used at concentrations below $30 \mathrm{ng} / \mathrm{ml}$. Similar results were obtained when CTL generated in a mixed lymphocyte reaction in the presence of IL-2 or IL-15 were tested for cytotoxicity against allogeneic target cells. IL-15 also stimulated the proliferation of human phytohemagglutinin (PHA)-activated $\mathrm{T}$ cells and their derivative $\mathrm{CD}^{+}$and $\mathrm{CD}^{+}$subsets (1).

IL-15 does not stimulate lymphocytes via the induction of IL-2 since a neutralizing IL-2 $\mathrm{mAb}$ does not inhibit the proliferative response of PHA-activated PBMC to IL-15 (1). Experiments with neutralizing mAbs suggest the participation of the IL-2Rß subunit but not the IL-2R $\alpha$ subunit in IL-15 binding and function. IL-2R $\alpha$ mAb could 
not inhibit IL-15-induced proliferation of human T cells, whereas antibodies to IL-2Rß (e.g., TU11, TU27, and Mik1ß) significantly inhibited all actions of IL-15 including the generation of CTL and LAK cells and the proliferation of PHA-activated human PBMC and murine CTLL.2 cells (1). It has been consistently demonstrated in subsequent studies that IL-15 must bind to IL-2Rß in order to initiate the signaling events which precede cellular activation $(2,6)$.

In order to determine whether the IL-2R $\gamma$ subunit is a component of the IL-15 receptor, COS-7 cells were transiently transfected with expression vectors for the IL-2Rß and IL-2R $\gamma$ chains, alone and in combination (7). COS-7 cells did not specifically bind IL-15 when transfected with either chain alone. However, a dramatic increase in binding of radiolabeled IL- 15 was observed when COS cells were co-transfected with both the IL-2Rß and IL-2R $\gamma$ subunits. Evidence for a physical interaction between IL-15 and the IL-2Rß and IL-2R $\gamma$ chains comes in the form of cross-linking experiments in which radiolabeled ligand was covalently linked to cell surface receptors. Cross-linking of radiolabeled IL-15 to IL-2Rß $\gamma$-transfected COS cells resulted in the formation of a high molecular weight complex which was consistent with the binding of IL-15 to surface IL-2Rß and IL-2R $\gamma$ subunits. Identical results were obtained when radiolabeled IL- 2 was crosslinked to IL-2Rß $\gamma$-expressing COS cells (7).

\section{The IL-15R $\alpha$ chain}

Initial studies by Grabstein et al. (1) suggested that IL-15 did not require the IL-2R $\alpha$ chain for binding or signal transduction. A search was undertaken for another receptor subunit which might possess high affinity binding for IL-15. Giri et al. (8) have recently reported the molecular cloning and characterization of the murine IL- $15 R \alpha$ chain. This molecule was isolated from a murine helper $\mathrm{T}$ cell line and is structurally related to the IL-2R $\alpha$ chain. Analysis of the cDNA clone reveals a type I membrane protein with a signal peptide of 32 amino acids, a 173-amino acid extracellular domain, a transmembrane domain of 21 amino acids, and a 37-amino acid cytoplasmic tail. Comparison of the murine IL-2R $\alpha$ and IL- $15 R \alpha$ proteins reveals the presence of a highly conserved protein-binding motif (the socalled "sushi domain"). The IL-15R $\alpha$ subunit has just one of these motifs in its extracellular region, whereas the murine IL-2R $\alpha$ protein has two such domains. The IL- $15 R \alpha$ subunit has a slightly longer cytoplasmic tail than the IL-2R $\alpha$ chain, but it does not exhibit any recognized catalytic or protein-binding domains, and it is unlikely that this receptor subunit is capable of transmitting an intracellular signal (8). IL-15R $\alpha$ transcript was detected in a macrophage line, a thymic stromal cell line, a bone marrow stromal cell, as well as in several $\mathrm{T}$ and $\mathrm{B}$ cell lines. In addition, IL-15R $\alpha$ mRNA was detected in a variety of normal tissues including spleen, heart, lung and skeletal muscle. Interestingly, the expression of mRNA for IL- $15 R \alpha$ was highest within murine liver. The expression of the IL-15R $\alpha$ chain appears to be augmented by activation signals in much the same manner as the IL-2R $\alpha$ subunit (8). Anderson et al. (9) have recently cloned and characterized the human IL-15R $\alpha$ subunit. Human IL-15R $\alpha$ possesses a single sushi domain. Transcript for IL-15R $\alpha$ was found to be present in peripheral blood monocytes and $T$ cells, as well as in several normal tissues including spleen, thymus, prostate, testis, ovary, and intestine. Recent work by our laboratory has revealed that freshly isolated human NK cells also express transcript for the IL-15R $\alpha$ chain (10).

A role for murine IL-15R $\alpha$ in the binding of IL- 15 was confirmed via experiments with a cell line which constitutively expresses the IL-2R $\gamma$ chain (8). Transfection of this cell 
line with IL-15R $\alpha$ and IL-2Rß reconstituted high affinity IL-15 binding as well as IL-15induced proliferation. Analysis of the binding of IL- 15 to the IL-15R $\alpha$ chain revealed that this receptor subunit has high affinity for IL-15 $\left(\mathrm{K}_{\mathrm{a}}=1.5 \times 10^{11}\right)$, but does not signal or prevent binding to IL-2Rßy. Thus, despite the similarity of their extracellular domains, the IL-2R $\alpha$ and IL-15R $\alpha$ protein subunits exhibited a 1000-fold difference in their affinity for their respective ligands (8). This finding is not predicted by our knowledge of the interaction between IL-2 and the IL-2R $\alpha$ subunit and suggests that the biological roles of IL-2 and IL-15 in vivo may be significantly different. This discovery, coupled with the widespread mRNA expression of IL-15R $\alpha$, suggests that IL-15 may have multiple physiological roles which are not shared with IL-2R $\alpha$. It will be important to determine whether the IL-15R $\alpha$ subunit can transmit an intracellular signal in tissues such as muscle which appear to lack expression of the IL-2Rß and IL-2R $\gamma$ chains. Alternatively, the IL-15R $\alpha$ chain may serve as a mechanism for sequestration of IL-15 or a means by which IL-15 is presented to other cells of the immune system.

Signaling

All evidence available to date suggests that the binding of IL- 15 to its receptor complex leads to a series of signaling events

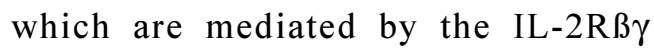
heterodimer (11). IL-15 signaling pathways should therefore be similar if not identical to those of IL-2 (11). Indeed, the functional response to IL-15 is closely similar to that of IL-2 for virtually all cell types examined to date $(2,6)$. However, some cells appear to respond preferentially to one cytokine or the other $(1,7)$. Such a differential response might be explained in terms of the binding affinity of each cytokine, which is governed, in turn, by receptor density and composition. Signal- ing events initiated by the binding of IL- 2 or IL-15 to the IL-2/15R have recently been reviewed (12).

Effects of IL-15 on cells of the immune system

T cells

IL-15 was originally discovered as a result of its ability to stimulate the proliferation of an IL-2-dependent murine T cell line (1). IL-15 can also stimulate the proliferation of PHA-activated peripheral blood $\mathrm{T}$ cells as well as their composite $\mathrm{CD}^{+}$and $\mathrm{CD}^{+}$subsets, and alloantigen-specific CTLs in mixed lymphocyte culture (1). More recently, IL-15 has been shown to stimulate the production of IL-5 in human T helper cell clones (13) and the growth of epidermal $\gamma \delta$ T cells by binding to the IL-2Rß $\gamma$ complex (14).

Wilkinson and Liew (15) have recently shown that IL-15 also exerts a chemoattractant effect upon $T$ cells. They found that both IL-15 and IL-2 could induce polarization of fresh and cultured human PBMC and they showed that the entire increase in polarized lymphocyte morphology could be attributed to the $\mathrm{T}$ cell compartment. $\mathrm{CD}^{+}$and $\mathrm{CD}^{+}$ cells polarized equally in response to IL-15 or IL-2. Lymphocytes cultured in the presence of $1 \mu \mathrm{g} / \mathrm{ml}$ IL- 15 exhibited significant active locomotion as compared to cells cultured in media alone and collagen gels containing IL-15 exhibited greater invasion of lymphocytes than did control gels. $\mathrm{T}$ cell locomotion could be enhanced by exposing cells to positive concentration gradients of IL-15 or by simply increasing the concentration of IL-15. These results imply that IL-15 can induce $\mathrm{T}$ cell movement as well as accelerate this movement. The authors hypothesized that accessory cells at inflammatory sites might attract $T$ cells to these areas via their production of IL- 15 . 
B cells

Armitage et al. (16) examined IL-15 for its ability to stimulate B cells in a series of in vitro activation assays and found that IL-15 is able to induce $\mathrm{B}$ cell proliferation and differentiation in much the same manner as IL-2. Neither IL-2 nor IL-15 could induce significant $B$ cell proliferation in the absence of co-stimulatory signals. However, purified human tonsillar B cells could proliferate in response to IL-15 if first activated with phorbol ester or anti-IgM-Ab. It has been previously shown that co-stimulation of $\mathrm{B}$ cells with IL-2 and CD40 ligand (CD40L) induces the production of significant amounts of immunoglobulin (16). IL-15 in combination with $\mathrm{CD} 40 \mathrm{~L}$ also stimulates the secretion of IgM, IgG, and IgA from B cells. Importantly, the combination of IL-2 and IL-15 had an additive effect upon B cell proliferation in this assay, which would imply that these two cytokines may act in concert to induce B cell immunoglobulin production after their stimulation with CD40L. Finally, the authors demonstrated that IL-15 is able to partially replace IL-2 in the induction of antigen-specific antibody production in vitro. These results demonstrate the ability of IL-15 to stimulate the differentiation and proliferation of human B cells and it is thus conceivable that IL-15 may play a role in $B$ cell activation in vivo, possibly in the absence of $\mathrm{T}$ cells.

NK cells

NK cells appear to be unique among lymphocytes in their constitutive expression of a functional IL-2R, but it is important to note that IL-2 is produced solely by T cells after stimulation with specific antigen. The high affinity IL-2R on activated T cells has 100 -fold greater affinity for IL-2 than the intermediate affinity IL-2Rß $\gamma$ expressed on the majority of NK cells. The activated T cell uses the IL-2 it secretes in an autocrine fashion to promote cell differentiation $(17,18)$. It has yet to be conclusively demonstrated that $\mathrm{T}$ cell-derived IL-2 acts as a stimulatory cytokine for NK cell function in vivo. In contrast, IL-15 is produced by activated monocytes and bone marrow stromal cells and may, therefore, be an important ligand for the NK cell IL-2R in vivo (4).

The majority of human NK cells express low levels of CD56 (>90\%) and an intermediate affinity IL-2Rß $\gamma$ which requires nanomolar concentrations (15-150 ng/ml) of IL-2 for activation and induces primarily a cytotoxic response (19). Incubation of CD56 dim NK cells with IL-15 results in significant LAK activity against NK-resistant cell lines, which is clearly dose-dependent (20). IL-15 and IL-2 induce nearly identical levels of cytotoxicity in CD56 ${ }^{\mathrm{dim}} \mathrm{NK}$ cells when used at equal concentrations, and both can be abrogated in the presence of an anti-IL-2RB $\mathrm{mAb}$. However, blockade of IL-2R $\alpha$ has no effect on the IL-15-induced cytolytic function of this NK cell subset (20). The majority of NK cells also express a low affinity receptor for the $F_{c}$ portion of immunoglobulin (CD16 or $\left.\mathrm{F}_{\mathrm{c}} \mathrm{R} \gamma \mathrm{III}\right)$ which allows them to interact with antibody-coated target cells and to mediate antibody-dependent cellular cytotoxicity (ADCC) (19) and IL-15 enhances ADCC in a dose-responsive fashion that is identical to that of IL-2 (20).

The expression of IL-15 protein by activated monocytes and bone marrow stromal cells and the constitutive expression of a functional IL-2/15R on human NK cells suggested that IL-15 might act as a survival factor for NK cells. To test this hypothesis, fresh NK cells were cultured in serum-free medium (SFM) in the presence or absence of IL-15. Cell viability was assessed by vital dye exclusion. IL-15 at concentrations as low as $0.1 \mathrm{ng} / \mathrm{ml}$ sustained NK cell survival over a period of 8 days, compared to no survival in SFM alone. NK cells cultured in 
Figure 1 - In vitro production of IFN- $\gamma$ by purified human natural killer cells. $\mathrm{CD}^{+} 6^{+}$natural killer cells were isolated from fresh blood by flow-activated cell sorting and cultured in medium supplemented with IL-12 (10 units $/ \mathrm{ml}$ ) and/or IL-15 (10 ng/ml). Culture supernatants were harvested at $72 \mathrm{~h}$ and analyzed for IFN- $\gamma$ by ELISA. Data are reported as mean \pm SEM for three replicates. This result is representative of five independent experiments.

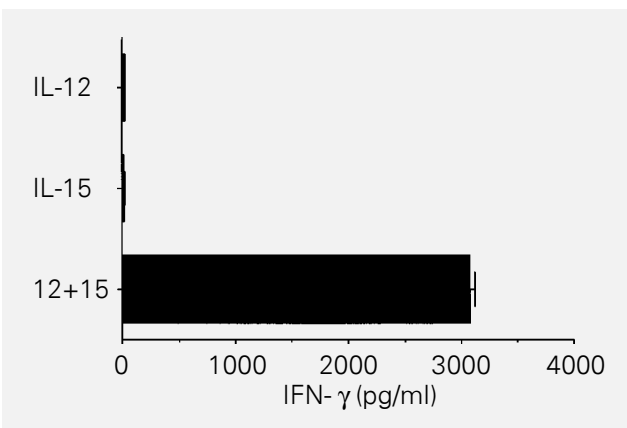

SFM underwent morphological changes and DNA cleavage consistent with programmed cell death within $48 \mathrm{~h}$. Using nuclear staining with propidium iodide, IL-15 was shown to exert a dose-dependent protective effect against DNA cleavage. NK cell survival was not sustained by other monocyte-derived factors (TNF- $\alpha$, IL-1 $1 \beta$, IL-10, or IL-12). Interestingly, NK cells cultured in SFM showed a rapid decline in Bcl-2 by immunoblot analysis, while addition of IL-15 maintained normal Bcl-2 levels. Thus, IL-15 is able to promote NK cell survival via the inhibition of programmed cell death and may regulate NK cell expression of Bcl-2 (10).

Given that IL-15 is produced by activated monocytes, we set about to determine if IL- 15 could act in an additive or synergistic manner with IL-12, a cytokine that is also stimulatory to NK cells and is also produced by activated monocytes. The combination of IL-12 and IL-15 produces additive to synergistic LAK activity that is nearly identical to that seen with IL-12 and IL-2. The combination of IL-2 and IL-15 did not result in an additive cytotoxic effect, which implies that the binding of these ligands to the IL-2Rß $\gamma$ results in the transduction of similar (if not identical) signals.

Another important effector function of the NK cell population is the production of cytokines which modulate the host immune response to infection. Specifically, NK cells can be stimulated in vitro and in vivo to produce relatively large quantities of IFN- $\gamma$, TNF- $\alpha$, GM-CSF, and MIP- $\alpha$, all of which are strong activators of monocyte function
(19). Several investigators have shown that IL-2 is required to stimulate maximal NK cell secretion of these cytokines (see below). However, the production of IL-2 by T cells appears to be temporally and spatially limited (18). The role of the monocyte in stimulating NK cell function takes on new importance in light of its ability to produce IL-15 $(1,4)$. We hypothesized that the monocyte can take part in NK cell activation via the secretion of IL-15, which would combine with IL-12 to induce the production of monocyte-stimulatory factors by the NK cell in a paracrine feedback mechanism $(21,22)$. We therefore investigated the production of IFN$\gamma$, TNF- $\alpha$, and GM-CSF by NK cells in response to IL-15, either alone or in combination with other monocyte-derived cytokines. NK cells were cultured under various conditions for $72 \mathrm{~h}$, at which time supernatants were harvested and analyzed by ELISA. The combination of IL-15 and IL-12 is a potent stimulus to the production of IFN- $\gamma$, TNF- $\alpha$, and GM-CSF by human resting natural killer cells (20). Figure 1 shows the results obtained for IFN- $\gamma$. Interestingly, TGF- $\beta$, another important cytokine product of monocytes, is a strong inhibitor of the IFN- $\gamma$ production of NK cells cultured with IL-15 and IL-12. Obviously, inhibitory cytokines such as IL-10 and TGF- $\beta$ may exert important regulatory effects upon NK cell cytokine secretion in vivo.

IFN- $\gamma$ production by NK cells is now known to be critical to the activation of host monocytes and the development of an effective innate immune response in the time period which precedes the antigen-specific $\mathrm{T}$ cell response (22). It has recently been shown in a number of in vitro and in vivo experimental systems that NK cell production of IFN- $\gamma$ is critical for the control of several intracellular pathogens $(21,22)$. This phenomenon has been best examined by studying monocytes infected with Listeria monocytogenes, Toxoplasma gondii, and Leishmania major (reviewed in Ref. 22). In 
the case of infection withListeria monocytogenes or Toxoplasma gondii, the production of both TNF- $\alpha$ and IL-12 by infected monocytes has been shown to be critical to the induction of IFN- $\gamma$ secretion by NK cells (23-25). This response was found to be markedly enhanced in the presence of exogenous IL-2 and at times was inefficient in the absence of IL-2 $(23,26)$. While NK cells constitutively express a functional IL-2 receptor (IL-2R) (19), neither monocytes nor NK cells make IL-2, which suggests that either IL-2 produced by antigen-activated $\mathrm{T}$ cells or other cytokines (such as IL-15) may also be important for the production of IFN- $\gamma$ by NK cells in vivo. We have shown that activated monocytes express abundant IL-15 protein by immunohistochemical staining and Western blot analysis (4). Moreover, priming of monocytes with IFN- $\gamma$ greatly enhances their ability to secrete IL-15 after stimulation with LPS (Marien M and Carson W, unpublished observations). Importantly, resting monocytes do not appear to secrete significant levels of IL-15 protein (4).

Activated monocytes produce IL-12 and IL-15 in response to activating stimuli and, in theory, these cytokines should stimulate NK cell production of IFN- $\gamma$. We sought to determine if co-culture of NK cells with LPS-activated monocytes would result in IFN- $\gamma$ production (4). Alone, neither monocytes nor NK cells produced detectable levels of IFN- $\gamma$ in either the presence or absence of LPS. Co-culture of unstimulated monocytes with unstimulated NK cells did not induce IFN- $\gamma$ production. However, when monocytes and NK cells were co-cultured in the presence of LPS, IFN- $\gamma$ was easily detectable in culture supernatants at $72 \mathrm{~h}$. Thus, LPS activation of human monocytes induces IFN- $\gamma$ production in NK cells. IFN- $\gamma$ was detected in culture supernatants $18 \mathrm{~h}$ after the addition of LPS, peaked in concentration at $48 \mathrm{~h}$, and underwent a significant decline by $96 \mathrm{~h}$ (4).

The kinetics and quantity of IL-15 pro- duction in this co-culture system were determined by ELISA and compared to IL-12 protein production in the same culture. As expected, significant levels of IL-15 and IL-12 were produced by NK/monocyte cocultures within $24 \mathrm{~h}$ of LPS activation. We next investigated whether endogenous IL-15 production by LPS-activated human monocytes was contributing to IFN- $\gamma$ secretion by human NK cells. Co-cultures of LPS-activated monocytes and NK cells were treated with neutralizing anti-IL-15 antiserum or a control antiserum. Culture in the presence of neutralizing anti-IL-15 antiserum resulted in a $77 \%$ decrease in IFN- $\gamma$ production as compared to a control antiserum (Figure 2). IFN$\gamma$ production could also be partially inhibited in the presence of an anti-IL-2Rß $\mathrm{mAb}(68 \%$ reduction), while an anti-IL-2 neutralizing antiserum had no significant effect. It is noteworthy that, while endogenous IL- 15 is required for optimal IFN- $\gamma$ production, complete abrogation was never achieved with higher concentrations of anti-IL-15 antiserum or anti-IL-2Rß mAb (4). This suggests that suboptimal amounts of IFN- $\gamma$ can be produced by NK cells in the presence of other monocyte-derived cytokines such as TNF- $\alpha$ and IL-12 (23-25). In fact, complete neutralization of IL-12 alone reproducibly abolished IFN- $\gamma$ production in this co-culture system. This work suggests that IL-15 is an important monocyte-derived cofactor for optimal production of IFN- $\gamma$ by human NK cells. As such, it may have a significant role

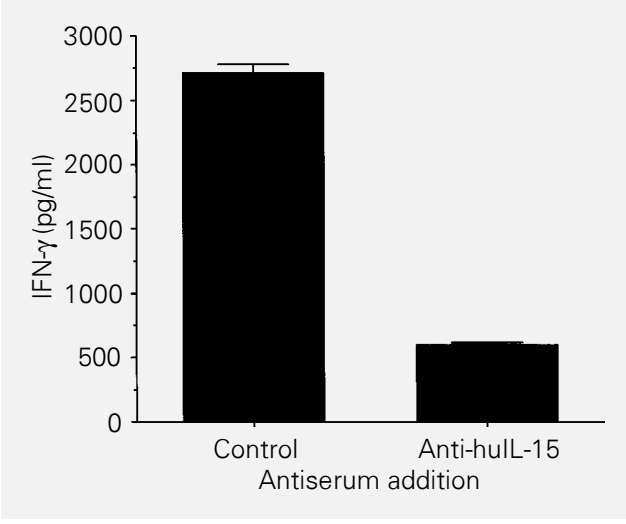

Figure 2 - Endogenous production of human IL-15 (hulL-15) is required for optimal production of IFN- $\gamma$. Co-cultures of $1 \times 10^{5}$ monocytes and $1 \times 10^{5} \mathrm{NK}$ cells were activated with lipopolysaccharide $(5 \mu \mathrm{g} / \mathrm{ml})$. The addition of anti-hulL-15 antiserum to co-cultures resulted in a $77.2 \pm$ $4.1 \%$ reduction in NK cell IFN- $\gamma$ production as compared to control. Data are reported as mean \pm SEM for three replicates. This result is representative of $12 \mathrm{in}$ dependent experiments $(\mathrm{P}<$ 0.0005; Student $t$-test) 
in the innate immune response to certain infections in vivo. Its production by activated monocytes provides additional insight as to why NK cells constitutively express a functional IL-2R.

\section{Conclusions}

IL-15 is a novel cytokine which exhibits no sequence homology with IL-2, yet is able to activate cells bearing functional IL-2R. The binding of IL-15 to the IL-2R appears to be mediated by the $\beta$ and $\gamma$ subunits. The newly discovered IL- $15 \mathrm{R} \alpha$ chain is similar in structure to the IL-2R $\alpha$ subunit but has extremely high affinity for its ligand and is expressed in a wide variety of tissues includ- ing several lymphoid cell lines. Thus far, it is believed that signaling via IL-2Rß $\gamma$ in response to IL-15 involves activation of the same protein tyrosine kinases that have been implicated in IL-2 signaling. The actions of IL-15 on T cells, B cells, and NK cells are therefore closely similar to those of IL-2; however, subtle differences do exist which may be attributed to receptor density and composition. In addition, other unique receptors for IL-15 are now being identified. Human IL-15 is produced by activated monocytes along with IL-12 and appears to be a critical cofactor for optimal production of IFN- $\gamma$ and, as such, it may have an important role in the innate immune response to infection.

\section{References}

1. Grabstein KH, Eisenman J, Shanebeck K, Rauch C, Srinivasan S, Fung V, Beers C, Richardson J, Schoenborn MA, Ahdieh M, Johnson L, Alderson MR, Watson JD, Anderson DM \& Giri JG (1994). Cloning of a $T$ cell growth factor that interacts with the $B$ chain of the interleukin-2 receptor. Science, 264: 965-968.

2. Tagaya $Y$, Bamford RN, DeFilippis AP \& Waldmann TA (1996). IL-15: A pleiotropic cytokine with diverse receptor/signaling pathways whose expression is controlled at multiple levels. Immunity, 4: 329-336.

3. Anderson DM, Johnson L, Glaccum MB, Copeland NG, Gilbert DJ, Jenkins NA, Valentine V, Kirstein MN, Shapiro DN, Morris SW, Grabstein K \& Cosman D (1995). Chromosomal assignment and genomic structure of IL15. Genomics, 25: 701-706.

4. Carson WE, Ross ME, Baiocchi RA, Marien MJ, Boiani M, Grabstein K \& Caligiuri MA (1995). Endogenous production of interleukin 15 by activated human monocytes is critical for optimal production of interferon- $\gamma$ by natural killer cells in vitro. Journal of Clinical Investigation, 96: 2578-2582.

5. Mrozek $E$, Anderson $P$ \& Caligiuri MA (1996). The role of interleukin 15 in the development of human CD56+ natural killer cells from CD34+ hematopoietic progenitor cells. Blood, 87: 2632-2640.

6. Giri JG, Anderson DM, Kumaki S, Park LS, Grabstein K \& Cosman D (1995). IL-15, a novel T cell growth factor that shares activities and receptor components with IL-2. Journal of Leukocyte Biology, 57: 763-766

7. Giri JG, Ahdieh M, Eisenman J, Shanebeck K, Grabstein K, Park LS, Cosman D \& Anderson D (1994). Utilization of the $\beta$ and $\gamma$ chains of the IL-2 receptor by the novel cytokine IL-15. EMBO Journal, 13: 2822-2830.

8. Giri JG, Kumaki $S$, Ahdieh M, Friend DJ, Loomis A, Shanebeck K, Dubose R, Cosman D, Park LS \& Anderson DM (1995). Identification and cloning of a novel IL-15 binding protein that is structurally related to the $\alpha$ chain of the IL-2 receptor. EMBO Journal, 14: 3654-3663.

9. Anderson D, Satoru K, Ahdieh M, Bertles J, Tometsko M, Loomis L, Giri J, Copeland NG, Gilbert DJ, Jenkins NA, Valentine V, Shapiro DN, Morris SW, Park LS \& Cosman D (1995). Functional characterization of the human interleukin-15 receptor $\alpha$ chain and close linkage of IL15RA and IL2RA genes. Journal of Biological Chemistry, 270: 29862-29869.

10. Carson WE, Lindemann MJ, Grabstein K \& Caligiuri MA (1997). A potential role of interleukin-15 in the regulation of human natural killer cell survival. Journal of Clinical Investigation, 99: 937-943.

11. Lin J-X, Migone T-S, Tsang M, Freidmann M, Weatherbee JA, Zhou L, Yamauchi A, Bloom ET, Meitz J, John S \& Leonard WJ
(1995). The role of shared receptor motifs and common Stat proteins in the generation of cytokine pleiotropy and redundancy by IL-2, IL-4, IL-7, IL-13, and IL-15. Immunity, 2: 331-339.

12. Taniguchi $T$ (1995). Cytokine signaling through nonreceptor protein kinases. Science, 268: 251-255.

13. Mori A, Suko M, Kaminuma O, Inoue $S$, Ohmura T, Nishizaki $Y$, Nagahori T, Asakura $Y$, Hoshino A, Okumura $Y$, Sato G, Ito K \& Okudaira H (1996). IL-15 promotes cytokine production of human $T$ helper cells. Journal of Immunology, 156: 2400-2405.

14. Edelbaum D, Mohamadzadeh M Bergstresser PR, Sugamura K \& Takashima A (1995). Interleukin (IL)-15 promotes the growth of murine epidermal $\gamma \delta T$ cells by a mechanism involving the $B$ and $\gamma_{c}$ chains of the IL-2 receptor. Journal of Investigative Dermatology, 105: 837-843.

15. Wilkinson PC \& Liew FY (1995). Chemoattraction of human blood T lymphocytes by interleukin-15. Journal of Experimental Medicine, 181: 1255-1259.

16. Armitage RJ, Macduff BM, Eisenman J, Paxton R \& Grabstein K (1995). IL-15 has stimulatory activity for the induction of $B$ cell proliferation and differentiation. Journal of Immunology, 154: 483-490.

17. Smith KA (1993). Lowest dose interleukin-2 immunotherapy. Blood, 81: 1414-1423. 
18. Smith KA (1988). Interleukin 2: Inception, impact, and implications. Science, 240: 1169-1176.

19. Robertson RJ \& Ritz J (1990). Biology and clinical relevance of human natural killer cells. Blood, 76: 2421-2438.

20. Carson WE, Giri JG, Lindemann MJ, Linett ML, Ahdieh M, Anderson D, Eisenmann J, Grabstein K \& Caligiuri MA (1994). Interleukin-15 is a novel cytokine which activates human natural killer cells via components of the interleukin-2 receptor. Journal of Experimental Medicine, 180: 1395-1403.

21. Biron CA \& Gazzinelli RT (1995). Effects of IL-12 on immune responses to microbial infections: A key mediator in regulating disease outcome. Current Opinion in
Immunology, 7: 485-496.

22. Bancroft GJ (1993). The role of natural killer cells in innate resistance to infection. Current Opinion in Immunology, 4: 503-510.

23. Tripp CS, Wolf SF \& Unanue ER (1993). Interleukin 12 and tumor necrosis factor $\alpha$ are costimulators of interferon $\gamma$ production by natural killer cells in severe combined immunodeficiency mice with listeriosis, and interleukin 10 is a physiologic antagonist. Proceedings of the National Academy of Sciences, USA, 90: 37253729.

24. Gazzinelli RT, Hieny S, Wynn TA, Wolf S \& Sher A (1993). Interleukin 12 is required for the T-lymphocyte-independent induction of interferon $\gamma$ by an intracellular para- site and induces resistance in T-celldeficient hosts. Proceedings of the $\mathrm{Na}$ tional Academy of Sciences, USA, 90: 6115-6119.

25. Hunter CA, Subauste CS, Vancleave VH \& Remington JS (1994). Production of gamma interferon by natural killer cells from Toxoplasma gondii-infected SCID mice. Regulation by interleukin-10, interleukin-12, and tumor necrosis factor alpha. Infection and Immunity, 62: 28182824.

26. Sharma SD, Hofflin JM \& Remington JS (1985). In vivo recombinant interleukin-2 administration enhances survival against a lethal challenge with Toxoplasma gondii. Journal of Immunology, 135: 4160-4163. 